\title{
The efficacy of ultrasound-guided multifidus cervicis plane block versus greater occipital nerve block for cervicogenic headache
}

\author{
Zenat Eldadamony Mohamed ${ }^{1}$, Carmen Ali Zarad ${ }^{2}$, Mohamed E. Flifel ${ }^{3}$ and Ali A. Abou Elmaaty ${ }^{4^{*}}$
}

\begin{abstract}
Introduction: Traditionally, non-invasive and invasive techniques were used for the treatment of cervicogenic headache $(\mathrm{CH})$. Greater occipital nerve block is the most frequent peripheral nerve block invasive technique used for the management of cervicogenic headache. The purpose of this prospective, double-blinded study was to compare the efficacy of two different techniques: multifidus cervicis plane block and greater occipital nerve block in the treatment of refractory cervicogenic headache by using ultrasound.

Methods: Sixty patients with cervicogenic headache were recruited and diagnosed according to the ICHD-III beta version. The patients were divided into two groups, one group was for greater occipital nerve block and the other group was for multifidus cervicis plane block with ultrasound-guided.

Results: Visual analog scale (VAS) was 2.09\% in the multifidus cervicis plane block (MCPB) group and was 2.22\% in the greater occipital nerve block (GONB) group with a median reduction of -4.33 and -3.048 , respectively, at 2week visits with a statistically significant difference better in the MCPB group $(P<0.001)$. At 4 weeks visits, VAS scale was better in the MCPB group than in the GONB group (3.79 and 4.44, respectively) with a median reduction in VAS scale ( -3.27 and -3.095 , respectively) and statistically significant differences between both groups $(P=0.020)$.

Conclusion: Both the ultrasound-guided multifidus cervicis plane block and greater occipital nerve block are effective as intervention techniques in the treatment of refractory cervicogenic headache. These techniques are simple, safe, more reliable with less side effects, and often reduce the requirements of analgesic drugs.
\end{abstract}

Keywords: Cervicogenic headache, Occipital nerve, Cervicis, Nerve block

\section{Introduction}

Headache is one of the most prevalent causes of chronic pain with its incidence of about $30 \%$ in adults [1].

In 2004, the International Headache Society classified headache into primary and secondary types in which cervicogenic headache $(\mathrm{CH})$ was considered a secondary type [2], while the International Classification of Headache Disorders, 3rd edition (beta version), reported the

\footnotetext{
* Correspondence: carmenali042@gmail.com

${ }^{4}$ Badr University Hospital, Helwan University, Helwan, Egypt

Full list of author information is available at the end of the article
}

diagnostic criteria of $\mathrm{CH}$ as follows: lesion or disorder in the soft tissue of the neck or cervical spine, pain was temporally related to the onset of the cervical disorder and improved after the treatment of the cervical problem, reduction in cervical range movement, worsening by provocation, and pain relief following diagnostic blocks [3].

Cervicogenic headache $(\mathrm{CH})$ is described as a chronic hemi-cranial pain resulted from a disorder of the cervical spine and its anatomic structures innervated by the C1, C2, and C3 cervical spinal nerves [4]. 
Many causative factors that may contribute to $\mathrm{CH}$ are degenerative, post-traumatic cervical spine pathology, cervical spine or muscle dysfunction, or pro-inflammatory cytokine release $[5,6]$. The prevalence of $\mathrm{CH}$ has been estimated at $0.7-13.8 \%$ according to different reports [7].

Traditionally, non-invasive therapeutic strategies (e.g., medication, physical activity, and manual manipulation) $[8,9]$ and invasive therapeutic strategies (e.g., radiofrequency [10, 11], acupuncture, and anesthetic block) [12] were the most preferred treatments by physicians. Regional anesthetic techniques, pulsed radiofrequency, or subcutaneous occipital nerve stimulation are considered minimally invasive procedures used for the treatment of refractory cervicogenic headache [13].

The greater occipital nerve (GON) is the sensory branch of the primary dorsal ramus of $\mathrm{C} 2$ with a possible contribution from the $\mathrm{C} 3$ dorsal ramus. It passes between $\mathrm{C} 1$ and $\mathrm{C} 2$ just below the posterior arch of the atlas and the lamina of the axis.

The ultrasound-guided technique was first described in a cadaveric study in 2010, and it was used selectively to block the GON at the $\mathrm{C} 2$ level [14]. GON block can be performed unilaterally or bilaterally, and it is indicated for the treatment of occipital neuralgia and different types of headache like cervicogenic headache, post-dural puncture headache, cluster type headache, or migraine [15]. GON is considered as the most frequent peripheral nerve block technique used for the management of cervicogenic headache [16].

Ohgoshi and his colleagues used a novel multifidus cervicis plane (MCP) block as perioperative analgesia for cervical laminoplasty. They achieved a good analgesic effect and concluded that MCP block may provide effective perioperative analgesia after cervical spine surgery [17].

\section{Objectives}

This prospective, double-blinded study was done to compare the efficacy of two different techniques: multifidus cervicis plane block and greater occipital nerve block in the treatment of cervicogenic headache using ultrasound.

\section{Methods}

This prospective study was approved by the Institutional Review Board (IRB), Faculty of Medicine (code number: R/19.04.483), and (the ClinicalTrials.gov identifier: NCT04454541) all patients had a written informed consent.

The study was performed in the period between May 2019 and December 2019 at out-patient clinics of the Neurology Department, University Hospital.

Sixty patients were included in this study. All 60 patients had cervicogenic headache disorders which were diagnosed according to the criteria of the International
Classification of Headache Disorder version III (ICHDIII beta version) [3].

This study included patients from both sexes, and patients' ages were more than or equal to 18 years old.

Younger age patients, patients not fulfilling the criteria of cervicogenic headache ICHD-III beta version, patients with a history of occipital nerve stimulation, patients with a history of occipital nerve injection, patients with a history of any occipital region surgical intervention, patients with a history of allergic reaction to any of materials used in the procedures, pregnant or lactating patients, patients with uncontrolled hypertension, patients with uncompensated congestive heart failure, patients with uncontrolled diabetes mellitus, patients with chronic liver disease, patients with chronic renal failure, patients with infectious and/or inflammatory diseases, patients with neoplasm and/or vascular disease, and patients with antiplatelet or anticoagulant therapy that may interfere with the procedure were excluded from this study.

Before the procedure, a full clinical neurological examination was performed on all patients. Routine laboratory investigations were done for all patients like renal function tests, liver function tests, complete blood count, and measurement of prothrombin time (PT) and activated partial thromboplastin time (APTT). Electrocardiography (ECG) was done for all patients. Measurement of saturation of pulse oxygen $\left(\mathrm{SpO}_{2}\right)$ was done for all patients.

Pain intensity was evaluated for all patients according to the visual analog scale (VAS) (0-10) (the pain VAS is a uni-dimensional, widely used scale in the measurement of the intensity of the pain comprising a horizontal (HVAS) or vertical (VVAS) line, usually $10 \mathrm{~cm}(100 \mathrm{~mm})$ in length) $[18,19]$.

Pain intensity evaluation was performed at the time of the initial diagnosis and performed later at the 2nd and 4th weeks after the procedures. The evaluation was performed by using the VAS for the side that affected by cervicogenic headache in a blinded manner.

Primary outcome headache-free interval $30 \mathrm{~min}$ after the procedure is described as a decrease of pain intensity about 30\% from the basal procedure level.

All procedures were performed using an ultrasound machine (Logiq. P5 pro model, GE brand, GE health care, France manufacture) with a high-frequency linear array transducer $(7-15 \mathrm{MHz})$; the US machine provided adequate penetration with a high-resolution image. The scanning was done in both transverse and longitudinal planes. Real-time ultrasound imaging was done by using both gray-scale and color Doppler techniques for accurate occipital artery localization.

\section{Interventional procedures}

Before the procedure, upper extremity venous access was established in all patients of both groups. Blood 
pressure (BP) and heart rate (HR) were measured for all patients. Electrocardiogram (ECG) was done for all patients, and pulse oxygen saturation $\left(\mathrm{SpO}_{2}\right)$ was also measured for all patients.

Before the procedure, all patients were infused with intravenous Ringer's solution $2 \mathrm{~mL} / \mathrm{kg} / \mathrm{h}$ and continuing low-flow of non-humidified oxygen $(2 \mathrm{~L} / \mathrm{min})$ with a nasal cannula. During the procedure, respiratory signs were monitored closely.

\section{Greater occipital nerve block}

The patient was lying in a prone position. For accurate localization of the occipital nerve, ultrasound-guided GONB was done by using a linear transducer that was sheathed in a sterile plastic package. To locate the occipital nerve, we searched first for the occipital artery in the medial one-third of the superior nuchal line in the area between the occipital tubercle and mastoid process by using both gray-scale and color Doppler ultrasound techniques (Fig. 1).

Before the injection, the scalp was cleaned with iodine, and the skin was sterilized.

In all patients, the occipital nerve was seen just medial to the occipital artery. Greater occipital nerve block was done by applying the injection just medial to the occipital artery by using a 22-gauge needle that was advanced beneath the lateral border of the ultrasound linear transducer with real-time ultrasound guidance and an inplane technique.

We detected the injected side according to the clinical symptoms of the patients and the painful side reported in the patients' headache complaint. During the procedure, we injected $10 \mathrm{ml}$ of $0.5 \%$ isobaric bupivacaine.

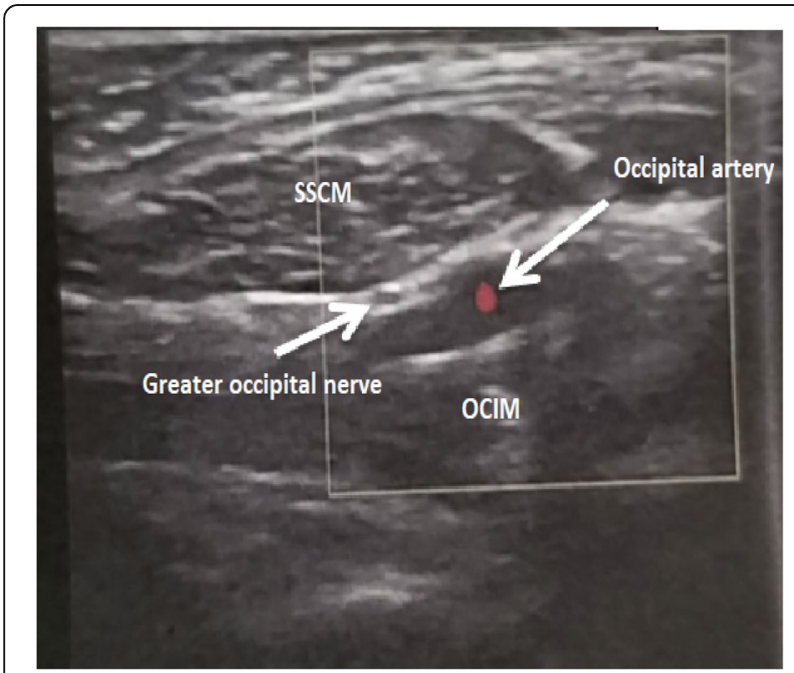

Fig. 1 Ultrasound imaging of the dorsal neck with color Doppler shows greater occipital nerve and occipital artery (white arrows), semispinalis capitis muscle (SSCM), and obliquus capitis inferior muscle (OCIM)
After injection, the patients were asked to lie down for $30 \mathrm{~min}$ to avoid dizziness.

\section{Multifidus cervicis plane block (MCPB) group}

The patient was asked to lie in a lateral position with the affected side directed upwards. Multiple gel cushions were placed under the patient's head, neck, and arm in order to make the patient's neck in a stable and mildly anterior flexion position. We determined the spinal level by the identification of the transverse process of the seventh and sixth cervical vertebrae (C7 and C6). The seventh cervical transverse process (C7) differs from the cervical vertebrae above by the presence of a rudimentary anterior tubercle and a prominent posterior tubercle. The transverse process of the sixth cervical spine with its characteristic sharp anterior tubercle could be visualized by moving the ultrasound transducer cranially; this allows for the easy identification of the consecutive cervical segments.

Before the procedure, all aseptic preparations of the injection area were done, and we used lidocaine $1 \%$ to anesthetize the skin.

The needle (22-G, $0.7 \mathrm{~mm} \times 60 \mathrm{~mm}$, Plexufx, BBRAUN, Tokyo, Japan) was introduced in-plane through the skin under continuous ultrasound guidance. The needle was advanced into the fascial plane between the semispinalis cervicis and multifidus cervicis muscles for the MCP block by injection of $20 \mathrm{ml}$ of $0.5 \%$ isobaric bupivacaine (Fig. 2).

After the procedure, all patients were continuously monitored by non-invasive monitoring $1 \mathrm{~h}$ before discharge to home.

\section{Informed consent}

All patients had a written consent before they were included in this study, and these consents were done after a complete explanation of the procedure steps, side effects, complications, and hoping benefits for all patients.

\section{Statistical analysis}

We used the clinical outcome variables for the intention-to-treat analysis. The data were presented as means and standard deviations (SD). The comparison between the studded groups was carried out using the $t$ test, analysis of variance (ANOVA) with the Tukey test, Honestly Significantly Different (HSD) test, CochranArmitage trend test, and Kruskal-Wallis test. Hommel's adjustments were carried out to correct the significance levels for multiple and post hoc testing. HodgesLehmann estimate of the median difference with a $95 \%$ confidence interval ( $95 \% \mathrm{CI}$ ) was used to determine the differences in the clinical variable outcome. The normality of variables was evaluated using the Shapiro-Wilk statistic. 


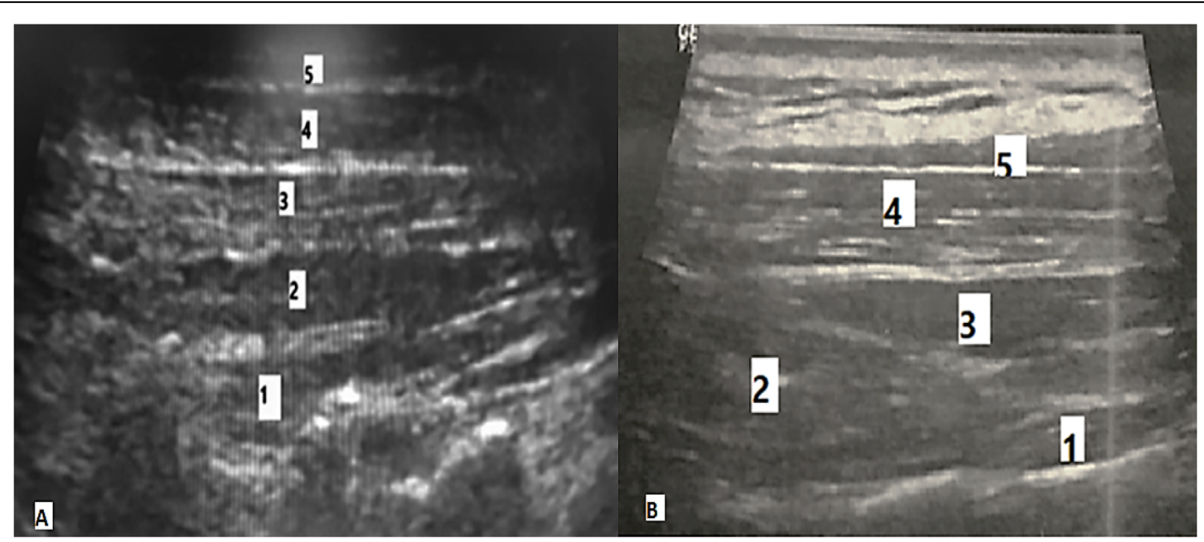

Fig. 2 a, b Ultrasound imaging of the dorsal neck muscles of two different patients show the (1) multifidus cervicis muscle, (2) semispinalis cervicis muscle, (3) semispinalis capitis muscle, (4) splenius muscle, and (5) trapezius muscle

The predictors of cervicogenic headache were determined by VAS through the conduction of a correlation analysis. Descriptive statistics, including frequency distributions for categorical variables, medians, and quartiles one and three (Q1, Q3) for continuous variables, were used for the characterization of the study sample at baseline assessment. Score changes between baseline and follow-up visits were assessed by comparing the frequency and median distributions at each time point, in the overall samples, and stratified by whether or not participants were deemed responders. For all analyses, $P$ values are two-sided, and the significance level was set at 0.05 . The $\alpha$ level was set at 0.05 for all tests.

A prior G-power analysis was done using the results obtained from previous studies and assuming alpha error of 0.05 and beta error of 0.2 (power of the study $80 \%$ ). A sample size of 30 patients per each group was calculated.

\section{Results}

This study included 60 patients with cervicogenic headache (30 patients received MCP injection, and 30 received GONB). Baseline characteristics, duration, and the side of headache did not differ significantly between the groups (Table 1).

The number of headache-free patients after $30 \mathrm{~min}$ of injection was larger in the MCPB group than in the GONB group without statistically significant differences between both groups $(P=0.248)$. The baseline VAS pain intensity was similar in both groups without statistically significant differences $(P=0.703)$. The VAS pain intensity after 2 and 4 weeks was lower in both groups with statistically significant differences $(P=0.001$ and 0.020 , respectively) (Table 2 ).

Headache frequency after 2 and 4 weeks was similar in both groups without statistically significant differences (Table 3).

\section{Discussion}

Cervicogenic headache is considered one of the most common types of headache worldwide. There are many lines of treatment for cervicogenic headache either noninvasive methods (pharmacotherapy, physiotherapy, etc.) or invasive therapeutic strategies (e.g., radiofrequency acupuncture, anesthetic block) $[9,12]$.

According to the underlying etiology of pain, there are many methods of local or regional anesthesia injections including nerve root injections, trigger point blocks, peripheral nerve blocks, or facet blocks $[9,12]$.

In our study, 60 patients with refractory cervicogenic headache were divided into two groups, each group consisted of 30 patients (GONB and MCPB groups).

We compared both groups regarding age, gender, BMI, duration of headache/weeks, and the side of headache (right/left), and there were no statistically significant differences between both groups.

Bovin and his colleagues and Inan and his colleagues studied the value of GONB and blocking of C2-C5 facet joint in the management of cervicogenic headache and found that simple GON blockade may be sufficient in many patients with cervicogenic headache, but little

Table 1 Demographic and clinical characteristics of patients

\begin{tabular}{llll}
\hline Variable & Group GONB & Group MCPB & $\boldsymbol{P}$ value \\
\hline Age (years), mean \pm SD & $41.1(10.0)$ & $41.9(11.3)$ & 0.402 \\
Height $(\mathrm{cm})$ & $160.7(6.3)$ & $167.4(8.2)$ & 0.708 \\
Weight & $68.2(25)$ & $71.2(15)$ & 0.990 \\
Gender, M/F & $3 / 27$ & $5 / 25$ & 0.442 \\
Body mass index (kg/h) & $25.8(3.5)$ & $24.6(3.9)$ & 0.074 \\
Duration of headache (weeks) & $19.9(12.8)$ & $17.9(12.2)$ & 0.094 \\
Side of the headache left/right & $14 / 16$ & $15 / 15$ & 0.0876 \\
\hline
\end{tabular}

All data are presented as number $(N), \%$, or mean \pm SD

$P \leq 0.05$ is significant

GONB Greater occipital nerve block, MCP Multifidus cervicis plane block 
Table 2 Headache intensity presented as VAS (primary outcome)

\begin{tabular}{llll}
\hline & GONB group & MCPB group & $P$ value \\
\hline -Post-intervention & & & 0.248 \\
-Headache-free 30 min & $14 / 30$ & $24 / 30$ & 0.703 \\
-Number of case percent & $45.5 \%$ & $62.5 \%$ & $\mathbf{0 . 0 0 1}$ \\
-Baseline VAS & $7.7(1.6)$ & $7.9(1.9)$ & $95 \% \mathrm{Cl}-1.85(-2.83$ to -0.86$)$ \\
-2 weeks VAS & $3.69(2.22)$ & $2.49(2.09)$ & $\mathbf{0 . 0 2 0}$ \\
-Mean reduction in VAS after 2 weeks & $3.048(2.60)$ & $4.33(1.9)$ & $95 \% \mathrm{Cl}-1.23(-2.26$ to -0.020$)$ \\
-4 weeks VAS & $4.44(2.23)$ & $3.79(2.57)$ & $3.27(2.36)$
\end{tabular}

GONB Greater occipital nerve block, MCP Multifidus cervicis plane block, VAS Visual analog scale

$P \leq 0.05$ is significant

response occurred in the case of $\mathrm{C} 3, \mathrm{C} 4$, and $\mathrm{C} 5$ isolated nerve blockade. Effective relief of pain was obtained by a combination of both $\mathrm{C} 2$ and $\mathrm{C} 3$ blockade, and partial effective relief of pain was obtained in the case of isolated C2 or C3 facet joint blockade [20, 21].

In our study, according to the baseline VAS, there are no statistically significant differences between both groups while at second and fourth week visits, there were statistically significant differences. This prolonged effect was matched with Sjaastad and his colleagues that revealed a prolonged effect lasts not only for days, but for weeks with a recommendation for utilizing it therapeutically [22].

Gabrhelik and his colleagues [13] concluded that pain and analgesic consumption significantly decreased in refractory cervicogenic headache with pulsed radiofrequency therapy technique to the greater occipital nerve using a mixture of local anesthetic and steroids after 3 months.

As regards headache frequency in both groups, there was a decrease in headache days per week but with no statistically significant differences in both groups $(P=$ 0.843 and 0.193 , respectively).

Many literatures described uncommon destructive treatment techniques like radiofrequency cervical zygapophyseal joint neurotomy, and C2 and or C3 ganglionectomies. These techniques may be complicated by neuroma formation and may cause subsequent hyperpathia and allodynia and all invariably cause sensory loss [11]. Our study concluded that the use of ultrasound-

Table 3 Headache frequency (secondary outcome)

\begin{tabular}{llll}
\hline & GONB group & MCPB group & $\boldsymbol{P}$ value \\
\hline Baseline frequency (no./week) & $6.7(5.2)$ & $5.5(4.3)$ & 0.216 \\
$\begin{array}{l}\text { 2-week visit frequency } \\
\text { (no./week) }\end{array}$ & $2.12(2.30)$ & $2.15(2.16)$ & 0.843 \\
$\begin{array}{l}\text { 4-week visit frequency } \\
\text { (no./week) }\end{array}$ & $2.36(2.40)$ & $2.04(2.09)$ & 0.193 \\
\hline
\end{tabular}

GONB Greater occipital nerve block, MCP Multifidus cervicis plane block $P \leq 0.05$ is significant guided GONB and MCPB in the treatment of refractory cervicogenic headache is considered as an alternative to these procedures with less side effects, more reliable, and simple technique.

\section{Conclusion}

Both ultrasound-guided MCPB and GONB techniques are effective intervention techniques used in the treatment of refractory $\mathrm{CH}$. Ultrasound-guided GONB and $\mathrm{MCPB}$ are simple, safe, have less side effects, more reliable and often reduce the requirements of analgesic drugs.

\section{Abbreviations}

APTT: Activated partial thromboplastin time; BP: Blood pressure; $\mathrm{CH}$ : Cervicogenic headache; $\mathrm{CL}$ : Confidence interval;

ECG: Electrocardiography; GONB: Greater occipital nerve block; HR: Heart rate; HSD: Honestly Significantly Different; ICHD: International Classification of Headache Disorder; IRB: Institutional Review Board; MCPB: Multifidus cervicis plane block; PT: Prothrombin time; IQR: Interquartile ranges; SD: Standard deviation; VAS: Visual analog scale

\section{Acknowledgements}

The authors are grateful to all patients and control subjects for their willingness to participate in this study.

\section{Authors' contributions}

ZEM, CAZ, MEF, and AAM all carried out the work. ZEM perform the procedure and did the statistical analysis. AAM was responsible for collecting the scientific data and writing the initial draft of the manuscript. MEF designs the protocol share in collecting the scientific data. CAZ performed the ultrasound of all patients. The authors read and approved the final version to be published.

\section{Funding}

There is no source of funding for the research.

\section{Availability of data and materials}

The data supporting the results of this article are included within the article.

\section{Ethics approval and consent to participate}

The authors obtained permission to conduct this study that was approved by the Institutional Review Board (IRB), Mansoura Faculty of Medicine, Mansoura University, Cairo, Egypt (code number: R/19.04.483), and (the ClinicalTrials.gov identifier: NCT04454541) all participants gave written informed consent. The procedures followed were in accordance with our protocol. The study was carried out during the period from May 2019 to December 2019 at the neurology outpatient clinic of Mansoura University Hospital. 


\section{Consent for publication}

Not applicable.

\section{Competing interests}

The authors declare that they have no competing interests.

\section{Author details}

${ }^{1}$ Mansoura University, Mansoura, Egypt. ${ }^{2}$ Port Said University, Port Fuad, Egypt. ${ }^{3}$ Mansoura University, Mansoura University, Mansoura, Egypt. ${ }^{4}$ Badr University Hospital, Helwan University, Helwan, Egypt.

Received: 13 April 2020 Accepted: 17 December 2020

Published online: 14 January 2021

\section{References}

1. Dočekal P, Keller O, Marková J, Opavský J. Bolesti hlavy. In: Rokyta R, Kršiak M, Kozák J, editors. Bolest. Praha: Tigis; 2006. p. 461-77.

2. International Headache Society. The international classification of headache disorders, 2nd edition. Cephalalgia. 2004;24(1):1-160.

3. Headache Classification Committee of the International Headache Society (IHS). The international classification of headache disorders, 3rd edition. Cephalalgia. 2018;38(1):1-211.

4. Fredriksen TA, Antonaci F, Sjaastad O. Cervicogenic headache: too important to be left un-diagnosed. J Headache Pain. 2015;16:6.

5. Chaibi A, Russell MB. Manual therapies for cervicogenic headache: a systematic review. J Headache Pain. 2012;13:351-9.

6. Martelletti P, Van Suijlekom H. Cervicogenic headache: practical approaches to therapy. CNS Drugs. 2004;18:793-805.

7. Vanelderen P, Lataster A, Levy R, Mekhail N, van Kleef M, Van Zundert Occipital neuralgia - review. Pain Pract. 2010;10:137-44.

8. Vincent MB. Headache and neck. Curr Pain Headache Rep. 2011;15(4):32431.

9. Jull G, Trott P, Potter H, Zito G, Niere K, Shirley D, et al. A randomized controlled trial of exercise and manipulative therapy for cervicogenic headache. Spine. 2002;27(17):1835-43.

10. Mehnert MJ, Freedman MK. Update on the role of z-joint injection and radiofrequency neurotomy for cervicogenic headache. PM R. 2013;5(3):2217.

11. Zhang J, Shi DS, Wang R. Pulsed radiofrequency of the second cervical ganglion (C2) for the treatment of cervicogenic headache. J Headache Pain. 2011;12(5):569-71.

12. Zipfel J, Kastler A, Tatu L, Behr J, Kechidi R, Kastler B. Ultrasound-guided intermediate site greater occipital nerve infiltration: a technical feasibility study. Pain Physician. 2016;19(7):E1027-34.

13. Gabrhelík T, Michálek P, Adamus. Pulsed radiofrequency therapy versus greater occipital nerve block in the management of refractory cervicogenic headache - a pilot study. Prague Med Rep. 2011;112(4):279-87.

14. Greher M, Moriggl B, Curatolo M, Kirchmair L, Eichenberger U. Sonographic visualization and ultrasound - guided blockade of the greater occipital nerve: a comparison of two selective techniques confirmed by anatomical dissection. Br J Anaesth. 2010;104:637-42.

15. Afridi SK, Shields KG, Bhola R, Goadsby PJ. Greater occipital nerve injection in primary headache syndromes-prolonged effects from a single injection. Pain. 2006;122:126-9.

16. Ashkenazi A, Levin M. Greater occipital nerve block for migraine and other headaches: is it useful? Curr Pain Headache Rep. 2007;11:231-5.

17. Ohgoshi Y, Izawa H, Kori S, Matsukawa M. Multifidus cervicis plane block is effective for cervical spine surgery. Can J Anesth. 2017;64:329-30.

18. McCormack HM, Horne DJ, Sheather S. Clinical applications of visual analogue scales: a critical review. Psychol Med. 1988;18:1007-19.

19. Jensen MP, Karoly P, Braver S. The measurement of clinical pain intensity: a comparison of six methods. Pain. 1986;27:117-26.

20. Bovim G, Berg R, Dale LG. Cervicogenic headache, anesthetic blockades of cervical nerves (C2-C5) and facet joint (C2/C3). Pain. 1992;49:315-20.

21. Inan N, Ceyhan A, Inan L, Kavaklioglu O, Alptekin A, Unal N. C2/C3 nerve blocks and greater occipital nerve block in cervicogenic headache treatment. Funct Neurol. 2001;16(3):239-43.

22. Inan N, Ceyhan A, Inan L, Kavaklioglu O, Alptekin A, Unal N. C2/ C3 nerve block and greater occipital nerve block in cervicogenic headache treatment. Funct Neurol. 2001;16:239-43.

\section{Publisher's Note}

Springer Nature remains neutral with regard to jurisdictional claims in published maps and institutional affiliations.

\section{Submit your manuscript to a SpringerOpen ${ }^{\circ}$ journal and benefit from:}

- Convenient online submission

- Rigorous peer review

- Open access: articles freely available online

- High visibility within the field

- Retaining the copyright to your article

Submit your next manuscript at $\boldsymbol{\nabla}$ springeropen.com 